J. Shinoda

Nagoya Math. J.

Vol. 49 (1973), 117-125

\title{
SOME CONSEQUENCES OF MARTIN'S AXIOM AND THE NEGATION OF THE CONTINUUM HYPOTHESIS
}

\author{
JUICHI SHINODA*)
}

§ 0. W. Sierpisnki [3] demonstrated 82 propositions, called $\mathrm{C}_{1}-\mathrm{C}_{82}$, with the aid of the continuum hypothesis. D. A. Martin and R. M. Solovay remarked in [2] that 48 of these propositions followed from Martin's axiom (MA), 23 were refuted by $\mathrm{MA}+2^{\aleph_{0}}>\aleph_{1}$ and three were independent of $\mathrm{MA}+2^{\boldsymbol{\aleph}_{0}}>\boldsymbol{\aleph}_{1}$. But the relation of the remaining eight propositions to $\mathrm{MA}+2^{\mathrm{N}_{0}}>\boldsymbol{\aleph}_{1}$ has been unsettled.

In this paper, we shall show at least five of them $\left(\mathrm{C}_{8}, \mathrm{C}_{13}, \mathrm{C}_{61}, \mathrm{C}_{62}\right.$ and $\left.\mathrm{C}_{70}\right)$ are also refuted by $\mathrm{MA}+2^{\aleph_{0}}>\boldsymbol{\aleph}_{1}$.

The following table gives the relation of $\mathrm{C}_{1}-\mathrm{C}_{82}$ to $\mathrm{MA}+2^{\boldsymbol{\aleph}_{0}}>\boldsymbol{\aleph}_{1}$.

$\begin{array}{lllllllllll} & 0 & 1 & 2 & 3 & 4 & 5 & 6 & 7 & 8 & 9 \\ 10 & \times & \times & \times & \times * & \bigcirc & \times & \bigcirc & \bigcirc & \bigcirc & \bigcirc \\ 20 & \bigcirc & \bigcirc & \bigcirc & \bigcirc & \bigcirc & \bigcirc & \times & \times & \times & \times \\ 30 & \bigcirc & \bigcirc & \times & \times & \times & \bigcirc & \bigcirc & \bigcirc & \bigcirc & \bigcirc \\ 40 & \bigcirc & \bigcirc & \bigcirc & \bigcirc & \bigcirc & \bigcirc & \bigcirc & ? & ? & \bigcirc \\ 50 & \times & \times & \triangle & \bigcirc & \bigcirc & \bigcirc & \bigcirc & \bigcirc & \bigcirc & \bigcirc \\ 60 & \bigcirc & \times * & \times * & \bigcirc & \bigcirc & \times & \times & \times & \times & \times \\ 70 & \times * & \bigcirc & \bigcirc & \bigcirc & \bigcirc & \bigcirc & \bigcirc & \times & \triangle & \times \\ 80 & ? & \triangle & \bigcirc & & & & & & & \end{array}$

By $\bigcirc$, we denote the propositions following from MA, by $\times$ the propositions refuted by $\mathrm{MA}+2^{\boldsymbol{N}_{0}}>\boldsymbol{\aleph}_{1}$, by $\triangle$ the propositions independent of $\mathrm{MA}+2^{\boldsymbol{\aleph}_{0}}>\boldsymbol{\aleph}_{1}$ and by ? the propositions whose relation to $\mathrm{MA}+$ $2^{\boldsymbol{N}_{0}}>\boldsymbol{\aleph}_{1}$ we do not know about at present.

Let $\mathscr{P}=\langle P, \leq\rangle$ be a partially ordered set. A subset $X$ of $P$ is said to be dense in $\mathscr{P}$ if, for every $p \in P$, there is $q \in X$ such that $p \leq q$. If $\mathscr{F}$ is a collection of dense subsets of $P$, a subset $G$ of $P$ is said to be an $\mathscr{F}$-generic filter on $\mathscr{P}$ if $G$ has the following properties:

Received February 7, 1972. Revised August 24, 1972.

* The author wishes to express his gratitude to Professors T. Tugué and K. Namba for their valuable suggestions and encouragement. 
(1) if $p, q \in P, p \in G$ and $q \leq p$, then $q \in G$;

(2) if $p, q \in G$, then there is $r \in G$ such that $p \leq r$ and $q \leq r$;

(3) if $X \in \mathscr{F}$, then $X \cap G \neq 0$.

If $p, q \in P$, then $p$ and $q$ are said to be compatible if there is $r \in P$ such that $p \leq r$ and $q \leq r . \quad \mathscr{P}$ is said to have the countable chain condition if every collection of pairwise incompatible elements of $P$ is countable.

Martin's axiom (MA) is the following statement:

If $\mathscr{P}=\langle P, \leq\rangle$ is a partially ordered set having the countable chain condition and $\mathscr{F}$ is a collection of dense open subsets of $P$ of cardinality $<2^{\aleph_{0}}$, then there exists an $\mathscr{F}$-generic filter on $\mathscr{P}$.

§1. In this section, we shall show $\mathrm{C}_{8}, \mathrm{C}_{9}, \mathrm{C}_{61}$ and $\mathrm{C}_{62}$ are refuted by $\mathrm{MA}+\neg \mathrm{CH}$. From [2], we quote the following lemma.

Lemma 1. Let $A$ and $B$ be collections of subsets of $\omega$, each of cardinality $<2^{\mathrm{r}_{0}}$, such that if $x \in B$ and $K$ is a finite subset of $A$ then $x-\cup K$ is infinite. If we assume MA, then there exists a subset $t$ of $\omega$ such that $x \cap t$ is finite if $x \in A$ and infinite if $x \in B$.

Let ${ }^{\omega} \omega$ be the set of all functions from $\omega$ into $\omega$, (more generally, $x_{y}$ be the set of all functions from $x$ into $y$ ). Following Sierpinski [3], we define a partial ordering $<$ on ${ }^{\omega} \omega$ as follows:

$$
f<g \leftrightarrow(\exists k \in \omega)(\forall n \geq k)[f(n)<g(n)] .
$$

The following lemma is due to K. Kunen [1].

LEMma 2. Let $F$ be a subset of ${ }^{\omega} \omega$ of cardinality $<2^{\aleph_{0}}$. If we assume MA, then there exists $g \in{ }^{\omega} \omega$ such that if $f \in F$ then $f<g$.

From Lemma 2, we have the following proposition, which is the negation of $\mathrm{C}_{9}$.

Proposition 1 (Assume MA and $2^{\aleph_{0}}>\aleph_{1}$ ). Let $E$ be an uncountable subset of $\boldsymbol{R}$, the set of reals, and $\left\langle f_{n}: n \in \omega\right\rangle$ be a convergent sequence of functions from $E$ to $\boldsymbol{R}$. Then there exists an uncountable subset $N$ of $E$ such that $\left\langle f_{n}: n \in \omega\right\rangle$ is uniformly convergent on $N$.

Proof. We may assume $E$ is of cardinality $\boldsymbol{\aleph}_{1}$. Let $f$ be the limit of $\left\langle f_{n}: n \in \omega\right\rangle$. Then for any $x \in E$ and $m \in \omega$, there is $k \in \omega$ such that if $n \geq k$ then $\left|f_{n}(x)-f(x)\right|<1 / m+1$. Take such $k \in \omega$ and denote it by $\varphi_{x}(m)$. Then we can define $\boldsymbol{\aleph}_{1}$ functions $\varphi_{x}$ from $\omega$ into $\omega$. Using 
Lemma 2, we can find $\varphi \in{ }^{\omega} \omega$ such that $\varphi_{x}<\varphi$ for all $x \in E$. For each $x \in E$, let $k_{x}$ denote the least $k \in \omega$ such that $\varphi_{x}(m)<\varphi(m)$ for all $m \geq k$. Since $E$ is uncountable, there is $k \in \omega$ and an uncountable subset $N$ of $E$ such that if $x \in N$ then $k_{x}=k$. Then for any $x \in N$ and $m \geq k$, if $n \geq \varphi(m)$ then $\left|f_{n}(x)-f(x)\right|<1 / m+1$. This means $\left\langle f_{n}: n \in \omega\right\rangle$ converges uniformly to $f$ on $N$.

Since $\mathrm{C}_{8}$ and $\mathrm{C}_{9}$ are equivalent, $\mathrm{C}_{8}$ is also refuted by $\mathrm{MA}+2^{\boldsymbol{\aleph}_{0}}>\boldsymbol{\aleph}_{1}$.

Recall that an $F_{\sigma}$-set is the union of a countable family of closed sets and a $G_{\delta}$-set is the intersection of a countable family of open sets.

LEMMA 3. ${ }^{1)}$ Let $X$ be a separable metric space of cardinality $<2^{\mathrm{No}_{0}}$. If we assume MA, then every subset of $X$ is $F_{\sigma}$ and $G_{\delta}$ in $X$.

Proof. Let $D$ be any subset of $X$ and $\left\{B_{i}: i \in \omega\right\}$ be a basis for open sets of $X$ such that all $B_{i}$ are non-empty. For each $x \in X$, let $s_{x}=$ $\left\{i \in \omega: x \in B_{i}\right\}$. If we put $A=\left\{s_{x}: x \in X-D\right\}$ and $B=\left\{s_{y}: y \in D\right\}$, then $A$ and $B$ are of cardinality $2^{N_{0}}$. It is easily checked that if $y \in D$ and $x_{1}$, $\cdots, x_{n} \in X-D$ then $s_{y}-\left(s_{x_{1}} \cup \cdots \cup s_{x_{m}}\right)$ is infinite. By Lemma 1, we can find a subset $t$ of $\omega$ such that $s_{x} \cap t$ is finite if $x \in X-D$ and $s_{y} \cap t$ is infinite if $y \in D$. For each $n \in \omega$, let

$$
K_{n}=\bigcup_{\substack{i>n \\ i \in t}} B_{i} .
$$

And let $K=\bigcap_{n \in \omega} K_{n}$. Then $K$ is a $G_{\delta}$-set of $X$. In order to prove that $D$ is a $G_{\tilde{\delta}}$-set of $X$, it suffices to prove the following (1) and (2):

$$
\begin{gathered}
D \subseteq K \\
(X-D) \cap K=0 .
\end{gathered}
$$

Let $y$ be an arbitrary element of $D$ and $n \in \omega$. Since $t \cap s_{y}$ is infinite, there is $i \in t \cap s_{y}$ such that $i>n$. Then $y \in B_{i}$ and $B_{i} \subseteq K_{n}$, so $y \in K_{n}$. Since $y$ and $n$ are arbitrary, we have (1). Let $x$ be any element of $X-D$. Since $t \cap s_{x}$ is finite, there is $n \in \omega$ such that if $i \in t$ and $i>n$ then $i \notin s_{x}$. For such $n \in \omega$, we have $x \notin K_{n}$, and so $x \notin K$. Thus we have (2).

Replacing $D$ with $X-D$, we have that $X-D$ is a $G_{\sigma^{-}}$-set of $X$. Hence $D$ is an $F_{\sigma}$-set of $X$. Therefore $D$ is $F_{\sigma}$ and $G_{\sigma}$ in $X$.

\footnotetext{
1) This lemma is a slight generalization of that of J. Silver.
} 
The following proposition is the negation of $\mathrm{C}_{62}$.

Proposition 2. (Suppose MA and $2^{\aleph_{0}}>\aleph_{1}$ ). Let $E$ be any uncountable set of reals and $f$ be any function from $E$ into $\boldsymbol{R}$, the set of reals. Then there exists an uncountable subset $N$ of $E$ such that $f \uparrow N$, the restriction of $f$ to $N$, is continuous on $N$.

Proof. We may assume $E$ is of cardinality $\aleph_{1}$. Let $F$ be an arbitrary closed set in $\boldsymbol{R}$. Then, by Lemma $3, f^{-1}(F)$, the inverse image of $F$, is a $G_{\tilde{\delta}}$-set of $E$. Thus $f_{a}$ is Baire function of class $\leqq 1$. As is well-known, every Baire function of class $\leq 1$ whose range is a subset of $\boldsymbol{R}$ is the limit of a sequence of continuous functions. Let $\left\langle f_{n}: n \in \omega\right\rangle$ be a sequence of continuous functions from $E$ to $\boldsymbol{R}$ which converges to $f$. Then, by Proposition 1, there exists an uncountable subset $N$ of $E$ such that $\left\langle f_{n}: n \in \omega\right\rangle$ converges uniformly to $f$ on $N$. Since each $f_{n} \uparrow N$ is continuous on $N$, so is $f \uparrow N$.

This proposition implies the following proposition, which is the negation of $\mathrm{C}_{61}$.

Proposition 3. (Suppose MA and $2^{\boldsymbol{\aleph}_{0}}>\boldsymbol{\aleph}_{1}$ ). There is a subset $\boldsymbol{F}$ of ${ }^{\boldsymbol{R}} \boldsymbol{R}$ of cardinality $2^{\mathrm{N}}$ such that if $g \in{ }^{\boldsymbol{R}} \boldsymbol{R}$ then for some $f \in \boldsymbol{F}$ the set $\{x \in \boldsymbol{R}: f(x)=g(x)\}$ is uncountable.

Proof. Let $F$ be the set of Baire functions from $\boldsymbol{R}$ into $\boldsymbol{R}$. Then clearly, $F$ is of cardinality $2^{\aleph_{0}}$. By Proposition 2, if $g \in{ }^{R} R$, then there exists an uncountable subset $N$ of $\boldsymbol{R}$ such that $g\lceil N$ is continuous on $N$. The following is a well-known theorem.

Let $X$ be an arbitrary metric space, let $Y$ be a complete separable space and $A$ be a subset of $X$. Then every Baire function from $A$ to $Y$ can be extended to a Baire function from $X$ into $Y$.

Since $f \uparrow N$ is a Baire function on $N$, by this theorem, there exists $f \in F$ such that $f \uparrow N=g \uparrow N$. Thus the set $\{x \in \boldsymbol{R}: f(x)=g(x)\}$ includes $N$, and is uncountable.

§2. Let $[\omega]^{\aleph_{0}}$ denote the set of all infinite subsets of $\omega$. We define a relation $\subseteq \subseteq^{*}$ on $[\omega]^{\aleph_{0}}$ as follows:

$$
a \subseteq^{*} b \leftrightarrow a-b \text { is finite, where } a, b \in[\omega]^{N_{0}} \text {. }
$$

Intuitively $a \subseteq * b$ iff $a \subseteq b$ almost everywhere. 
Lemma. ${ }^{1)}$ Suppose MA. Let $\Theta$ be an ordinal such that $\Theta<2^{\aleph_{0}}$, and let $\left\langle a_{\alpha}: \alpha\langle\Theta\rangle\right.$ be a sequence of elements of $[\omega]^{\aleph_{0}}$ such that if $\alpha<\beta<\Theta$ then $a_{\beta} \subseteq{ }^{*} a_{\alpha}$. Then there exists $a \in[\omega]^{N_{0}}$ such that if $\alpha<\Theta$ then $a \subseteq{ }^{*} a_{\alpha}$.

Proof. Let $A=\left\{\omega-a_{\alpha}: \alpha<\Theta\right\}$ and $B=\left\{a_{\alpha}: \alpha<\Theta\right\}$. Then clearly, $A$ and $B$ are of cardinality $<2^{\aleph_{0}}$. If $\alpha, \alpha_{1}, \cdots, \alpha_{n}<\Theta$, then

$$
a_{\alpha}-\bigcup_{i=1}^{n}\left(\omega-\alpha_{\alpha_{i}}\right)=a_{\alpha} \cap a_{\alpha_{1}} \cap \cdots \cap a_{\alpha_{n}} .
$$

It is easily checked the intersection of finite elements of $B$ is an element of $[\omega]^{\aleph_{0}}$. Thus $A$ and $B$ satisfy the condition of Lemma 1 of $\S 1$. Therefore there is a subset a of $\omega$ such that $a-a_{\alpha}$ is finite and $a \cap a_{\alpha}$ is infinite for any $\alpha<\Theta$. For such $a \subseteq \omega$, we have $a \in[\omega]^{\aleph_{0}}$ and $a \subseteq a_{\alpha}$.

From this lemma, we obtain the following proposition, which is the negation of $\mathrm{C}_{13}$.

Proposition. (Assume MA and $2^{\boldsymbol{\aleph}_{0}}>\boldsymbol{\aleph}_{1}$ ). Let $\left\langle f_{n}: n \in \omega\right\rangle$ be $a$ sequence of functions from $\boldsymbol{R}$ to $\boldsymbol{R}$. Then there exists a sequence $\left\langle m_{k}: k \in \omega\right\rangle$ of natural numbers such that $m_{0}<m_{1}<\cdots<m_{k}<\cdots$ and the set $\left\{x \in \boldsymbol{R}:\left\langle f_{m_{k}}(x): k \in \omega\right\rangle\right.$ converges to a finite or infinite value $\}$ is uncountable.

Proof. ${ }^{2)}$ For each $a \in[\omega]^{\aleph_{0}}$, let $a^{\prime}$ denote the sequence $\left\langle n_{k}: k \in \omega\right\rangle$ such that $n_{0}<n_{1}<\cdots<n_{k}<\cdots<\cdots$ and $a=\left\{n_{k}: k \in \omega\right\}$. By the limit of the sequence $\left\langle f_{n}(x): n \in a\right\rangle$, we mean the limit of the sequence $\left\langle f_{n_{k}}(x): k \in \omega\right\rangle$ in the usual sense, where $\left\langle n_{k}: k \in \omega\right\rangle=\alpha^{\prime}$. Let $E$ be a subset of $\boldsymbol{R}$ of cardinality $\boldsymbol{\aleph}_{1}$. Order $E$ into a transfinite sequence of type $\omega_{1}$ as follows:

$$
x_{0}, x_{1}, \cdots, x_{\alpha}, \cdots \quad\left(\alpha<\omega_{1}\right)
$$

By transfinite induction on $\alpha$, we define a sequence $\left\langle a_{\alpha}: \alpha<\omega_{1}\right\rangle$ of elements of $[\omega]^{\aleph_{0}}$ such that $a_{\beta} \subseteq{ }^{*} a_{\alpha}$ if $\alpha<\beta<\omega_{1}$ and the sequences $\left\langle f_{n}\left(x_{\alpha}\right): n \in a_{\alpha}\right\rangle$ with $\alpha \in \omega_{1}$ are convergent. The sequence $\left\langle f_{n}\left(x_{0}\right): n \in \omega\right\rangle$ includes a convergent subsequence $\left\langle f_{n_{k}}\left(x_{0}\right): k \in \omega\right\rangle$, whose limit is finite or infinite. So, we define $a_{0}$ to be $\left\{n_{k}: k \in \omega\right\}$. Assume that $a_{\beta}$ with $\beta<\alpha$ are defined and $a_{r} \subseteq * a_{\beta}$ if $\beta<\gamma<\alpha$. Then, by the above lemma, we can find $a \in[\omega]^{\aleph_{0}}$ such that $a \subseteq{ }^{*} a_{\beta}$ for all $\beta<\alpha$. The sequence $\left\langle f_{i}\left(x_{\alpha}\right)\right.$ :

1) It was pointed out by the referee that this lemma could be proved from Lemma 2 of $\S 1$.

2) This proof was suggested to the author by Professor Kanji Namba. 
$i \in a\rangle$ includes a convergent subsequence $\left\langle f_{i_{k}}\left(x_{\alpha}\right): k \in \omega\right\rangle$. So, we define $a_{\alpha}$ to be $\left\{i_{k}: k \in \omega\right\}$.

By the lemma of this section, let $b$ be an element of $[\omega]^{\text {so }_{0}}$ such that $b \subseteq{ }^{*} a_{\alpha}$ for all $\alpha<\omega_{1}$. For every $\alpha<\omega_{1}$, since $b \subseteq{ }^{*} a_{\alpha}$, the sequence $\left\langle f_{m}\left(x_{\alpha}\right) ; m \in b\right\rangle$ is convergent. If we put $\left\langle m_{k}: k \in \omega\right\rangle=b^{\prime}$, then the set $\left\{x:\left\langle f_{m_{k}}(x): k \in \omega\right\rangle\right.$ is convergent $\}$ includes $E$, and is uncountable.

§3. Let $E$ be a subset of $\boldsymbol{R}$ and $a \in \boldsymbol{R}$. By $E(a)$ we denote the set $\{x+a: x \in E\}$.

Without MA, we can prove the following proposition.

Proposition. (Suppose $2^{\boldsymbol{\aleph}_{0}}>\boldsymbol{\aleph}_{1}$ ). If $E$ is an uncountable subset of $\boldsymbol{R}$ such that its complement is of cardinality $2^{\boldsymbol{N}_{0}}$, then there exists $a \in \boldsymbol{R}$ such that $E(a) \triangle E$, the symmetric difference of $E(a)$ and $E$, is uncountable.

Proof. Suppose, on the contrary, that for any $a \in R, E(a) \triangle E$ is countable. Let $N$ be a subset $E$ of cardinality $\boldsymbol{\aleph}_{1}$. Then we show $\bigcap_{x \in N}[\boldsymbol{R}-E(-x)] \neq 0$. If $\bigcap_{x \in N}[\boldsymbol{R}-E(-x)]=0$, then $\boldsymbol{R}=\cup_{x \in N} E(-x)$. On the other hand

$$
\bigcup_{x \in N} E(-x)=\bigcup_{x, y \in N}[E(-x) \triangle E(-y)] \cup \bigcap_{x \in N} E(-x) .
$$

Therefore,

$$
A \cup \bigcap_{x \in N} E(-x)=R \text {, where } A=\bigcup_{x, y \in N}[E(-x) \triangle E(-y)] .
$$

Since $A$ and $\bigcap_{x \in N} E(-x)$ are disjoint, we have $R-\bigcap_{x \in N} E(-x)=A$. Let $x$ be an arbitrary element of $N$. Then we have $R-E(-x) \subseteq A$. Note that each $E(a) \triangle E(b)$ is countable because $E(a) \triangle E(b)=J(a) \cup K(b)$, where $J=E(b-a) \triangle E, K=E(a-b) \triangle E$. Therefore $A$ is of cardinality $\leq \aleph_{1}$. This contradicts the hypothesis that the complement of $E$ is of cardinality $2^{\aleph_{0}}$. Thus $\bigcap_{x \in N}[R-E(-x)] \neq 0$.

Let $a \in \bigcap_{x \in N}[R-E(-x)]$, then $N \subseteq R-E(-a)$ because $a \notin E(-x)$ iff $x \notin E(-a)$. Therefore $E(-a) \triangle E$ includes $N$, and is uncountable.

The following corollary is the negation of $\mathrm{C}_{70}$.

COROLlaRY. (suppose MA and $2^{\aleph_{0}}>\aleph_{1}$ ). Let $E$ be a non-measurable set of reals. Then for some $a \in R, E(a) \triangle E$ is uncountable.

Proof. If we assume MA, then every set of reals of cardinalily 
$<2^{\aleph_{0}}$ is of Lebesgue measure $0([2, \S 4])$. Hence, if $E$ is non-measurable, the $E$ and its complement are of cardinality $2^{\mathrm{N}_{0}}$. Thus $E$ satisfies the condition of the proposition.

§4. A set $E$ of reals is said to have the property $(\boldsymbol{M})^{1)}$ if, for any collection $\mathscr{U}$ of open sets satisfying the condition

$$
(\forall x \in E)(\forall \varepsilon>0)(\exists U \in \mathscr{U})[\delta(U)<\varepsilon \wedge x \in U]
$$

where $\delta(U)$ is the diameter of $U$, there is a sequence $\left\langle U_{n}: n \in \omega\right\rangle$ of elements of $\mathscr{U}$ such that $E \subseteq \cup_{n \in \omega} U_{n}$ and $\lim _{n \rightarrow \infty} \delta\left(U_{n}\right)=0$.

As a direct application of MA, we have the following proposition.

Proposition. (Suppose MA). Every set of reals of cardinality $<2^{\aleph_{0}}$. has the property $(M)$.

Proof. Let $E$ be a set of reals of cardinality $<2^{\aleph_{0}}$, and $\mathscr{U}$ be a collection of open sets satisfying the condition (*). For each $n \in \omega$, there is a sequence $\left\langle U_{n m}: m \in \omega\right\rangle$ of elements of $\mathscr{U}$ such that $E \subseteq \cup_{m \in \omega} U_{n m}$ and $\delta\left(U_{n m}\right)<1 / n+1$ for all $m \in \omega$. We define a partially ordered set $\mathscr{P}=$ $\langle P, \leq\rangle$ as follows:

$$
\begin{aligned}
& P=\{p: p \text { is a finite function with } \operatorname{dom}(p) \cup \operatorname{rang}(p) \subseteq \omega\}, \\
& p \leq q \leftrightarrow p \subseteq q .
\end{aligned}
$$

Then clearly, $\mathscr{P}$ satisfies the countable chain condition. For each $x \in E$, if we put $X_{x}=\left\{p \in P: x \in \bigcup_{n \in \operatorname{dom}(p)} U_{n p(n)}\right\}$, then $X_{x}$ is dense in $\mathscr{P}$. Let $\mathscr{F}=\left\{X_{x}: x \in E\right\}$. Then $\mathscr{F}$ is of cardinality $<2^{\aleph_{0}}$, so there is an $\mathscr{F}$ generic filter $G$ on $\mathscr{P}$. If we put $f=\bigcup G$, then $f$ is a function with $\operatorname{dom}(f) \subseteq \omega$ and $\operatorname{rang}(f) \subseteq \omega$. We define $U_{n}$ as follows:

$$
U_{n}= \begin{cases}U_{n f(n)} & \text { if } n \in \operatorname{dom}(f) \\ U_{n 0} & \text { otherwise }\end{cases}
$$

Then, clearly, $U_{n} \in \mathscr{U}$ and $\lim _{n \rightarrow \infty} \delta\left(U_{n}\right)=0$. Let $x$ be an arbitrary element of $E$. Since $X_{x} \cap G \neq 0$, there is $p \in G$ such that $x \in \cup_{n \in \operatorname{dom}(p)} U_{n p(n)}$. Since $P \in G$, we have $\bigcup_{n \in \operatorname{dom}(p)} U_{n p(n)} \subseteq \bigcup_{n \in \omega} U_{n}$, so $x \in \cup_{n \in \omega} U_{n}$. Therefore $E$ has the property $(\boldsymbol{M})$.

1) See [3, p. 48] 
§5. A set $E$ of reals is said to have the property $(\lambda)^{1)}$ if every countable subset of $E$ is a $G_{\delta}$-set of $E$.

In this section, we shall show there is a non-measurable set of reals of cardinality $2^{\aleph_{0}}$ with the property $(\lambda)$.

$A$ set $E$ of reals is said to have the property $\left(S^{*}\right)^{2)}$ if, for every set $N$ of Lebesgue measure $0, E \cap N$ is of cardinality $<2^{\boldsymbol{N}_{0}}$. If a set $E$ is measurable and has positive measure, then $E$ includes a set of measure 0 and cardinality $2^{\boldsymbol{N}_{0}}$. If we assume MA, then every set of reals of cardinality $<2^{N_{0}}$ is of Lebesgue measure 0 . Therefore every set of reals of cardinality $2^{N_{0}}$ with the property $\left(S^{*}\right)$ is non-measurable. The existence of a non-measurable set of reals of cardinality $2^{\mathrm{s}_{0}}$ with the property $(\lambda)$ follows from the following proposition.

Proposition. (Suppose MA). There is a set $E$ of reals of cardinality $2^{\aleph_{0}}$ with the property $\left(S^{*}\right)$ such that every subset of $E$ of cardinality $<$ $2^{\aleph_{0}}$ is $G_{\delta}$ in $E$.

Proof. Order the set of all $G_{\dot{\delta}}$-sets of measure 0 into a transfinite isequence of type $2^{\text {sol }_{0}}$ as follows:

$$
N_{0}, N_{1}, \cdots, N_{\xi}, \cdots,\left(\xi<2^{\aleph_{0}}\right) \text {. }
$$

By transfinite induction on $\alpha$, we define a sequence $\left\langle x_{\alpha}: \alpha<2^{\aleph_{0}}\right\rangle$ of reals and a sequence $\left\langle K_{\alpha}: \alpha\left\langle 2^{\aleph_{0}}\right\rangle\right.$ of $G_{\delta}$-sets of measure 0 . Let $K_{0}=N_{0}$ and $x_{0}$ be an arbitrary element of $\boldsymbol{R}$. Suppose $x_{\beta}$ and $K_{\beta}$ with $\beta<\alpha$ are defined, and let

$$
S_{\alpha}=\bigcup_{\beta<\alpha} K_{\beta} \cup\left\{x_{\beta}: \beta<\alpha\right\} \cup N_{\alpha} .
$$

Then, by MA, $S_{\alpha}$ is of measure 0 , so $R-S_{\alpha} \neq 0$. Let $x_{\alpha}$ be an arbitrary element of $R-S_{\alpha}$ and $K_{\alpha}$ be the first $N_{\xi}$ such that $S_{\alpha} \cup\left\{x_{\alpha}\right\} \subseteq N_{\xi}$.

Let $E$ be the set $\left\{x_{\alpha}: \alpha<2^{\aleph_{0}}\right\}$. Then we have

(1) $E$ is of cardinality $2^{\aleph_{0}}$;

(2) for each $\alpha<2^{\mathrm{\aleph}_{0}}, E \cap N_{\alpha}$ is of cardinality $<2^{\mathrm{N}_{0}}$;

(3) $K_{\alpha} \subseteq K_{\beta}$ if $\alpha<\beta<2^{\aleph_{0}}$.

From (1) and (2), $E$ is a set of cardinality $2^{\boldsymbol{s}_{0}}$ with the property $\left(S^{*}\right)$.

Let $D$ be an arbitrary subset of $E$ of cardinality $<2^{\mathrm{N}_{0}}$. Since $2^{\mathrm{s}_{0}}$ is a regular cardinal, there is $\alpha<2^{\text {so }}$ such that $D \subseteq\left\{x_{\beta}: \beta \leq \alpha\right\}$. Put

\footnotetext{
1) See $[3$, p. 94]

2) Cf. $[3$, p. 81]
} 
$X=\left\{x_{\beta}: \beta \leq \alpha\right\}$. Then, by Lemma 3 of $\S 1, D$ is a $G_{i}$-set in $X$. Since $X=E \cap K_{\alpha}$ and $K_{\alpha}$ is $G_{\delta}$ in $R, X$ is $G_{\dot{\delta}}$ in $E$. Therefore $D$ is a $G_{\delta}$-set in $E$.

\section{REFERENCES}

[1] K. Kunen, Inaccessibility properties of cardinals, Doctoral Dissertation, Stanford University, 1968.

[2] D. A. Martin and R. M. Solovay, Internal Cohen extensions, Annals of Math. Logic vol. 2 (1970) 143-178.

[ 3 ] W. Sierpinski, Hypothèse du contnu, Second Edition (Chelsea, New York, 1956).

Nagoya University 